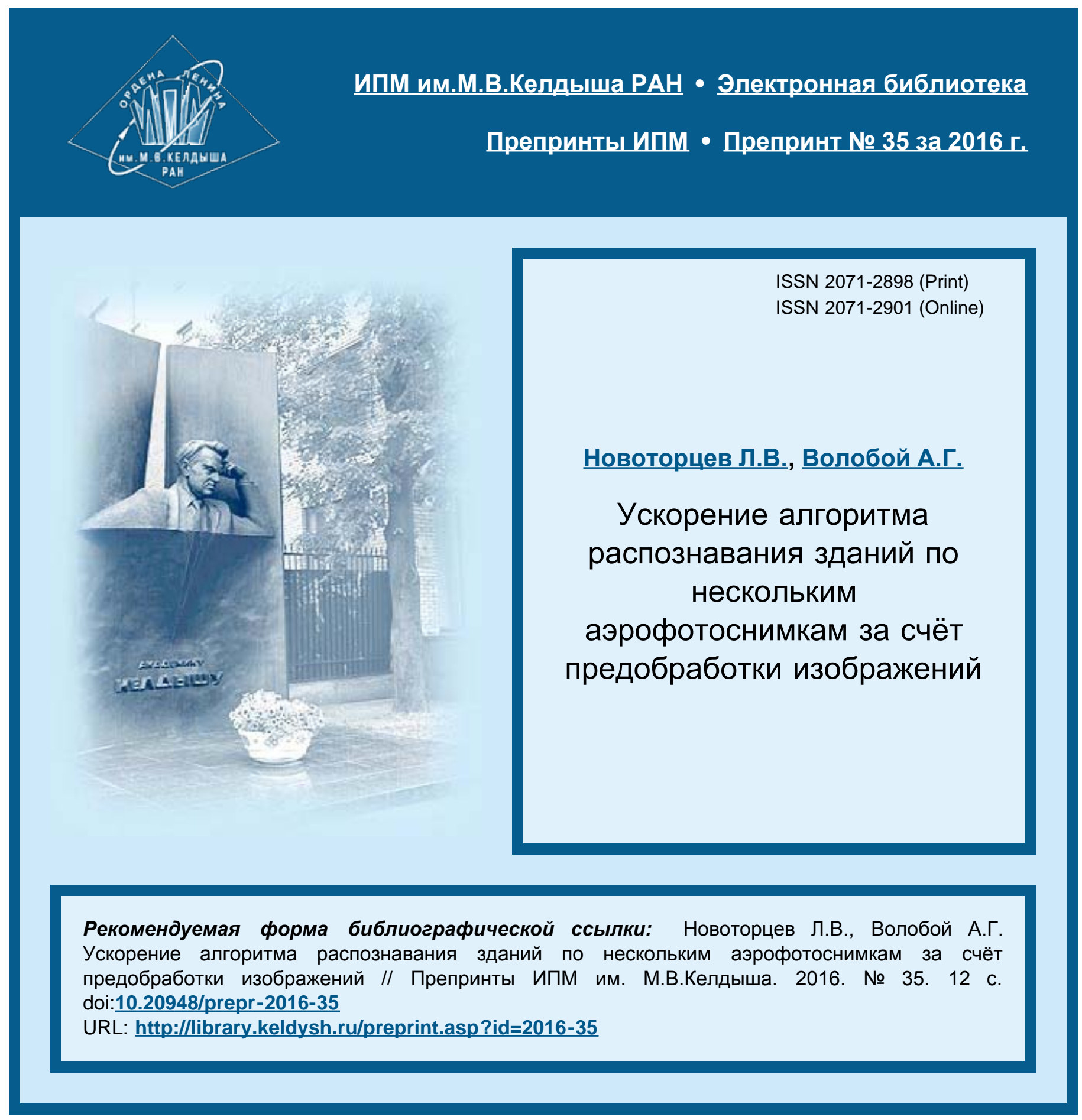




$$
\begin{gathered}
\text { О р д е н } \text { Л е н и н а } \\
\text { ИНСТИТУТ ПРИКЛАДНОЙ МАТЕМАТИКИ } \\
\text { имени М.В.Келдыша } \\
\text { Р о с и й с о й а к а е м и н а у к }
\end{gathered}
$$

Л.В. Новоторцев, А.Г. Волобой

\section{Ускорение алгоритма} распознавания зданий

по нескольким аэрофотоснимкам за счёт предобработки изображений 


\section{Новоторцев Л.В., Волобой А.Г.}

Ускорение алгоритма распознавания зданий по нескольким аэрофотоснимкам за счёт предобработки изображений

В данной работе рассматривается задача нахождения и реконструкции строений на аэрофотоснимках с большим пересечением. Стандартные методы решения этой задачи построены на основе сопоставления отрезков по всем изображениям. Такая операция занимает много времени. Поэтому предлагается использовать предобработку изображений, основанную на анализе взаимного расположения отрезков. Таким образом, основному алгоритму необходимо будет обработать гораздо меньшее количество данных для достижения результата.

Ключевые слова: компьютерное зрение, обнаружение прямых, обнаружение зданий, аэрофотоснимки, предобработка

Novotortsev L.V., Voloboy A.G.

Acceleration of multi image building recognition algorithm using image preprocessing

In the paper the task of detection and reconstruction of buildings on aero images is described. Standard methods, which are based on line matching, are very time consuming. We propose to do an image preprocessing by analyzing straight line geometry before applying one of standard methods. In this way a main algorithm will process much less data and finally building reconstruction will be done faster.

Key words: computer vision, line detection, building recognition, aero images, preprocessing

\section{Оглавление}

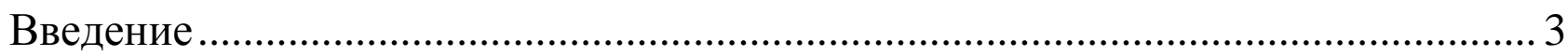

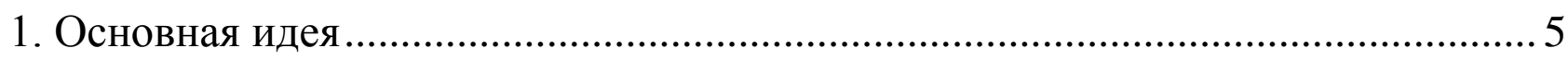

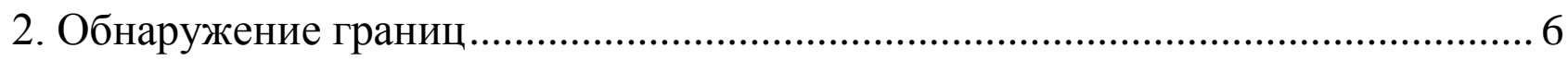

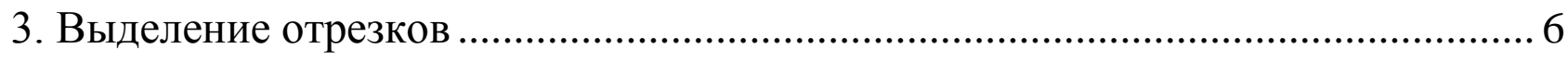

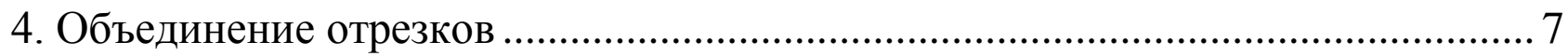

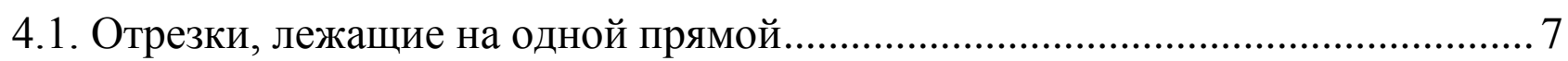

4.2. Отрезки, лежащие на пересекающихся прямых …………................................ 8

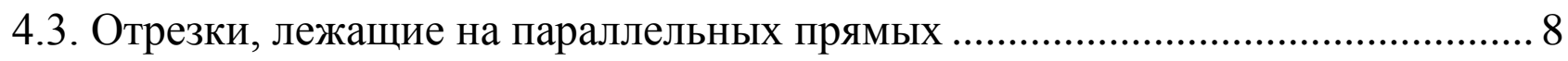

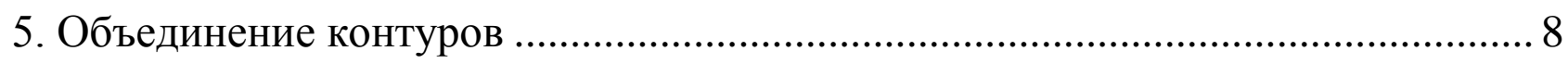

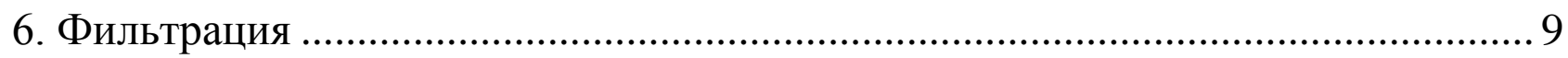

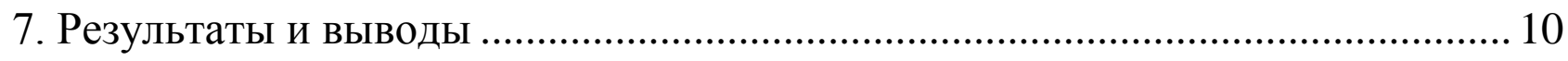

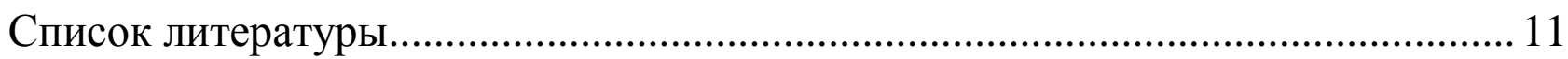




\section{Введение}

Последнее время человек активно осваивает и занимает новые территории, а также активно модернизирует и изменяет застройку и инфраструктуру уже освоенных земель. Это вызывает необходимость проводить регулярный оперативный анализ территории для построения актуальных карт, обнаружения застроек, линий электропередач, а также контроля вырубки леса.

Для решения этих задач на сегодняшний день целесообразнее всего использовать аэро- и космосъемку. Особенно это актуально для стран, обладающих большой территорией, где проводить подобные операции с земли невозможно.

Задачей же обработки и анализа полученных снимков (определением формы, размера, положения и иных характеристик снимков и объектов на них) занимается наука фотограмметрия. Одной из подзадач фотограмметрии является обнаружение и реконструкция (получение размеров и других параметров) строений по полученным снимкам. Это используется для решения таких задач, как построение карт GIS, построение моделей городов и их развития, обнаружение незаконных или опасных застроек. На данный момент есть несколько методов решения подобных задач.

Фотограмметрия занимается определением формы, размера положения и иных характеристик объектов, присутствующих на фотоснимках. Одним из основных направлений этой дисциплины является анализ снимков, сделанных с воздуха (аэрофотосъёмка, съёмка с беспилотных летательных аппаратов) и с орбиты (космосъёмка). Наиболее распространена на данный момент аэрофотосъемка за счёт высокого качества и доступности. Данное направление имеет широкий спектр применения: создание топографических карт, геологические изыскания, проектирование и строительство различных сооружений, автоматизированное построение пространственных моделей объекта по снимкам.

На сегодняшний день существует несколько подходов к решению данной задачи.

Первый подход ориентирован на построение плотной ЦММ (Цифровая модель местности). ЦММ представляет собой матрицу с данными о высоте земной поверхности, включая здания, растительность и другие высотные объекты. После ЦММ обрабатывается с целью обнаружения и реконструкции зданий. Обобщённые методы выделения изображений [1], [2], [3] дают довольно неточные результаты. По этой причине часто применяются методы, использующие априорную информацию об искомых объектах. К примеру, существует метод, ориентированный на обнаружение только плоских крыш [4].

Второй подход основан на использовании структурной, контекстной и спектральной информации об изображении [5], [6], [7], [8]. В данном подходе предполагается использование для обнаружения зданий только одного изображения. Для достижения результата используются алгоритмы 
обнаружения контуров, в основном применяются различные модификации фильтров Собеля [9] и Кэнни [10], метода Хафа [11]. Также используется анализ цветности и яркости отдельных элементов для ещё большего увеличения точности. В некоторых подходах также предлагается находить и проводить анализ теней. Такие подходы могут давать высокую точность. Однако такие методы всё равно уступают по точности методам, опирающимся на анализ нескольких изображений.

Третий подход связан с обработкой данных, полученных при помощи специальных методов, таких как LiDAR (световое обнаружение и определение дальности). Алгоритмы, основанные на таком подходе, [12] и [13], дают высокий процент обнаружения объектов и низкую вероятность ложного срабатывания, однако во многих случаях подобные специальные данные недоступны.

Последний подход, представляющий для нас наибольший интерес, связан с обнаружением линейных черт на различных изображениях, последующим сопоставлением этих черт и построением трёхмерной модели на основе результатов сопоставления [14]. Такой подход обеспечивает хорошие результаты за счёт сбора информации с нескольких изображений. Но он довольно долго обрабатывает данные потому, что необходимо выполнить сопоставление. Данный недостаток можно компенсировать, ограничив область каждого изображения, которую обрабатывает алгоритм.

В данной статье будет рассматриваться задача ускорения обнаружения строений по аэрофотоснимкам. Снимки, полученные таким образом, обладают рядом свойств, которые значительно облегчают их анализ. Пример такого снимка приведён на рис. 1.

Во-первых, фотографии, снятые с самолетов, имеют высокое качество за счёт того, что на самолётах можно размещать большие и тяжёлые камеры (большие габариты и вес позволяют увеличить отношение качества к цене). Данное свойство также уменьшает стоимость отдельного снимка. Во-вторых, направление съёмки почти не отличается от надирного (вертикального направления). Возможные отклонения значительно меньше градуса, и их можно не учитывать. В-третьих, съёмка ведётся маршрутами. Это означает, что самолёт делает множество снимков за один пролёт над интересующей областью и изображения, сделанные на одном маршруте, имеют малое отличие по времени между собой, что исключает значительные изменения в области съёмки. Обычно каждая точка маршрута покрывается двумя-четырьмя снимками, что позволяет применять вышеописанный алгоритм даже при наличии только одного маршрута. Обычно их всё же несколько и они имеют пересечения между собой, что увеличивает количество снимков каждой из точек области. 


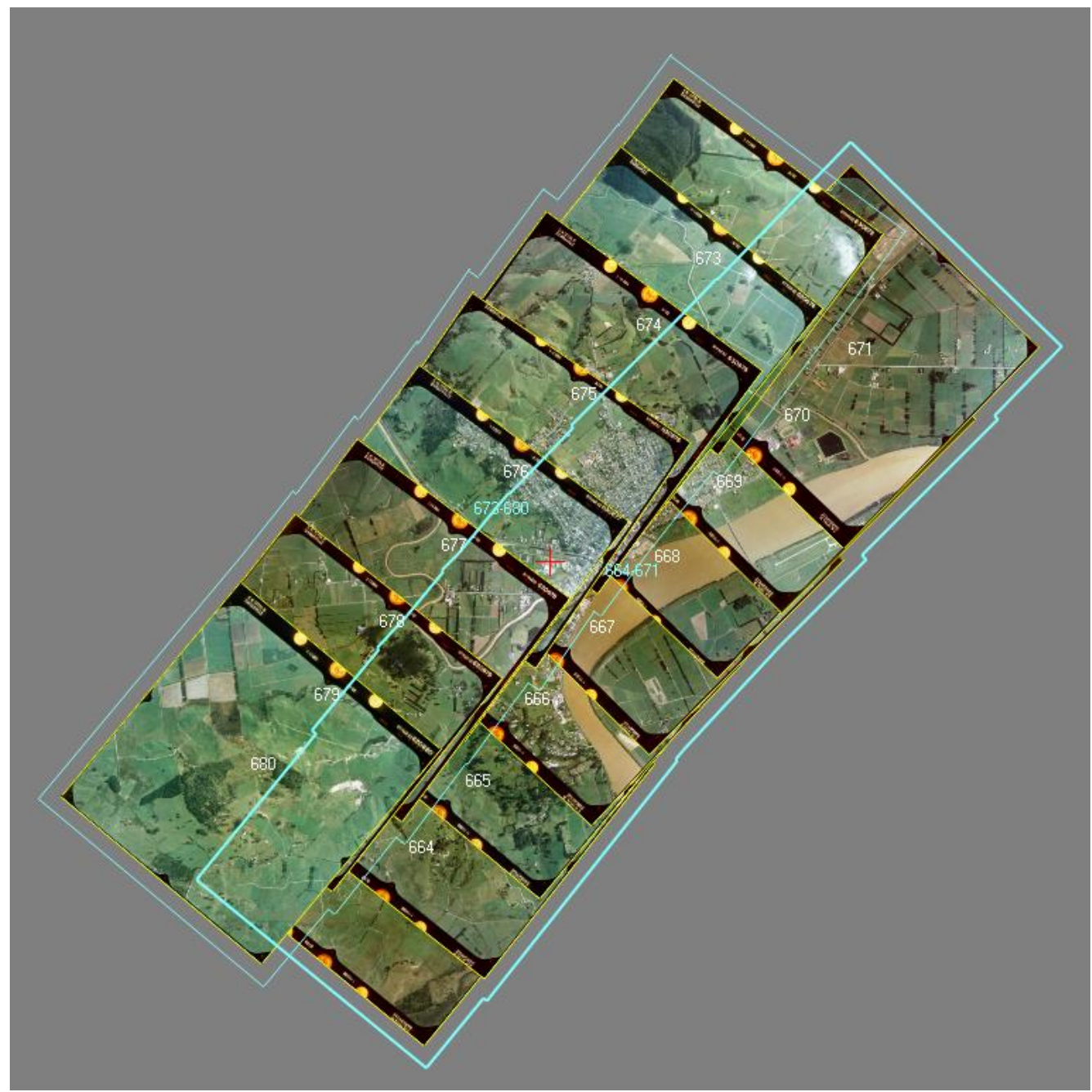

Рис. 1. Пример аэрофотосъёмки

Основная идея алгоритма, представленного в данной статье, заключается в обнаружении отрезков на изображении и их последующем объединении в замкнутые контуры. Области, которые попали в эти контуры, далее будут обрабатываться более подробным и точным алгоритмом. Это позволит значительно ускорить процесс выделения крыш за счёт уменьшения площади, подвергающейся точной, ресурсоёмкой обработке.

Кроме того, большое количество «точных» алгоритмов используют данные о границах, которые вычисляются в приведённом методе. Таким образом, алгоритм, описанный в данной статье, может существенно снизить время обработки аэрофотоснимков.

\section{1. Основная идея}

В данной статье мы предлагаем использовать тот факт, что большинство строений имеют линейные черты. По этой причине мы выбрали метод выделения отрезков на изображении в качестве основы предложенного метода. Использование этой особенности позволит увеличить точность определения 
необходимых объектов и облегчит вычисления. Весь алгоритм может быть разделён на следующие подзадачи:

1. обнаружение границ;

2. выделение прямых линий и отрезков;

3. объединение отрезков в замкнутые контуры;

4. отбраковка заведомо ложных контуров.

\section{2. Обнаружение границ}

Первым шагом представленного алгоритма является обнаружение границ на изображении. Основным подходом к решению этой задачи является построение карты градиентов яркости изображения в каждой точке и использование значений вычисленных градиентов в качестве показателя границы. На этом подходе основывается фильтр Собеля [9]. В тех случаях, когда задача обнаружения границ подразумевает двоичное решение (есть граница в точке или нет границы в точке), используется фильтр Кэнни [10]. Этот метод, помимо того, что производит бинаризацию границы, ещё и выполняет такие операции, как сужение границы.

\section{3. Выделение отрезков}

Далее по полученной карте границ производятся векторизация и обнаружение отрезков. Наиболее распространённым методом решения этой задачи является алгоритм Хафа [11]. Основная идея заключается в том, чтобы построить дискретную матрицу, в которой каждая ячейка соответствует прямой: строка и колонка соответствуют длине нормали и углу её наклона. Далее для каждой точки, помеченной как граница на предыдущем этапе, производятся следующие вычисления:

1. для каждого из значений угла наклона из таблицы вычисляется длина нормали;

2. для всех пар «угол-длина» в соответствующей ячейке матрицы инкрементируется её значение.

Из заполненной матрицы выделяются ячейки, содержащие значения, являющиеся локальными максимумами. Значения угла и длины нормали берутся как параметры найденной прямой.

Из-за дискретизации параметров в процессе поиска прямых методом Хафа может возникнуть погрешность. Изучению этой проблемы и методов её решения посвящено немало научных работ: [15], [16], [17], [18].

В данной работе для решения этой задачи предлагается использовать следующий подход. Вначале мы разделяем изображение на равные квадратные области (за исключением границ изображения). Для каждой области применяется модификация метода Хафа, оперирующая с окрестностями отрезков [15]. Отрезки, найденные на соседних сегментах, объединяются, если они лежат на одной прямой. 
После применения такого подхода мы получим набор отрезков, являющихся частью линейных объектов на изображении. Эти отрезки необходимо объединить для получения единых контуров.

\section{4. Объединение отрезков}

Для решения этой задачи было решено использовать два входных параметра для алгоритма слияния отрезков. Первый параметр - «ожидаемое расстояние между строениями» (ОРМС). Если расстояние между отрезками меньше этого значения, то считается, что они принадлежат одному объекту сцены. Вторым параметром является «ожидаемый размер строений» (ОРС). Если расстояние больше этого значения, то предполагается, что несвязанные отрезки принадлежат разным объектам. Возможность вычислять данные параметры автоматически будет исследована в дальнейших работах.

Далее поочерёдно рассматриваются пары ближайших друг другу отрезков и решается, надо ли их соединять. Существует три варианта взаимного расположения отрезков, каждый из которых обрабатывается отдельно:

1. отрезки лежат на одной прямой;

2. отрезки лежат на пересекающихся прямых;

3. отрезки лежат на параллельных прямых.

После того как все отрезки рассмотрены, проводится замыкание получившихся ломаных линий. Рассмотрим эти операции подробнее.

\section{1. Отрезки, лежащие на одной прямой}

Иногда отрезок, соответствующий одной из сторон здания, прерывается. Это может быть связано с плохим качеством изображения, тенями, перекрывающим объектом или малым контрастом. Необходимо иметь возможность восстанавливать такие разрывы.

Использование для этих целей порогового значения недостаточно эффективно, поскольку величина разрывов может сильно варьироваться. В данной статье предлагается подход, основанный на использовании двух пороговых значений (ОРМС и ОРС).

Если при рассмотрении двух отрезков расстояние между ними меньше, чем ОРМС, то эти отрезки сливаются в один. Если расстояние больше, чем ОРС, и больше каждого из отрезков, то отрезки не соединяются. Если не выполнено ни одно из этих условий, то производится более детальное изучение области между отрезками.

Вначале для этой области выполняется локальное растяжение яркости. Потом используется алгоритм Кэнни. После этого считается количество граничных пикселей, попавших на отрезок между рассматриваемыми отрезками. Если полученное число незначительно отличается от предполагаемой длины дополнительного отрезка, то рассматриваемые отрезки сливаются в один. 


\section{2. Отрезки, лежащие на пересекающихся прямых}

Если два отрезка находятся на пересекающихся прямых, то для каждого из них возможны два варианта положения точки пересечения.

Первый вариант - точка пересечения находится на самом отрезке. В этом случае отрезок разделяется на два точкой пересечения.

Второй вариант - точка пересечения находится на продолжении отрезка. Рассмотрение этого варианта идентично рассмотрению случая с отрезками на одной прямой за тем исключением, что анализу подлежит не расстояние между отрезками, а расстояние от конца отрезка до точки пересечения.

\section{3. Отрезки, лежащие на параллельных прямых}

При таком расположении отрезков имеет смысл рассматривать два варианта взаимного расположения отрезков.

В первом случае отрезки сдвинуты относительно друг друга и ровно одна пара концов отрезков находится близко друг к другу. В этом случае предполагается, что, возможно, пропущен сегмент, соединяющий эти отрезки. Для этого случая предлагается провести проверку, описанную в разделе 4.1 для отрезка, соединяющего ближайшие концы рассматриваемых отрезков. Если проверка прошла, то они соединяются.

Во втором случае отрезки мало отличаются по длине. При этом их концы находятся на небольшом расстоянии друг от друга. Эта ситуация характерна для случаев, когда у объекта на изображении есть двойная граница. В этом варианте рассматривается расстояние между отрезками. Если оно незначительно (не больше чем $10 \%$ от ОРС) и если один из отрезков является частью контура, то тогда контур расширяется до второго отрезка. В противном случае всё остаётся как есть.

Отрезки, не принадлежащие ни одному из типов, просто пропускаются и не участвуют в дальнейших вычислениях в рамках предложенного метода.

\section{5. Объединение контуров}

На предыдущем этапе мы получили набор ломаных линий, которые могут и не быть замкнутыми. Поэтому их необходимо замкнуть, чтобы получить замкнутый контур. Отрезок, построенный для замыкания ломаной, будем называть «замыкающим». На данном этапе ломаные линии могут получиться разветвлёнными и иметь больше двух концов. Поскольку мы выполняем фактически предобработку данных, то гораздо важнее не допустить пропуск объектов, чем выделение лишнего. Поэтому соединяться будут все концы, соединение которых не приведёт к пересечению замыкающего отрезка с ломаной. После этого проводится повторный анализ замыкающих отрезков.

Если некоторые замыкающие отрезки имеют пересечения друг с другом (согласно условию построения они не могут пересекаться с другими частями ломаной), то они разбиваются точкой пересечения на разные отрезки (каждый 
из них тоже считается «замыкающим»). После этого удаляются те замыкающие отрезки, что оказались внутри контуров.

В результате данной операции мы получаем набор контуров, которые ограничивают зоны, где могут находиться здания. В дальнейшем более аккуратный алгоритм будет обрабатывать только эти зоны.

\section{6. Фильтрация}

Скорость реконструкции крыш зависит как от общей площади выделенных областей, так и от количества этих областей. Таким образом, наличие большого количества маленьких областей может привести к существенному увеличению времени, затрачиваемого на работу непосредственно алгоритма обнаружения. Это противоречит основной цели данного метода.

По этой причине необходимо отфильтровать области, заведомо не включающие строения и, как следствие, крыши. Единственные критерии отбраковки, доступные на данном этапе, - это размеры областей и их форма.

Отбраковывать по форме получившейся области некорректно. Это связанно с тем, что рассмотренный алгоритм довольно прост из-за использования исключительно геометрических соображений при построении контуров. По этой причине контуры могут включать в себя, помимо строений, ещё и произвольные области вокруг них. Это приводит к тому, что контуры могут иметь практически любые формы.

По аналогичной причине ограничение площади и линейных размеров сверху тоже не является допустимым.

Единственный критерий, по которому можно провести фильтрацию, - это ограничение размеров областей снизу. В случае если область получилась четырехугольной, данное ограничение можно выразить в виде ограничения на длину каждой из сторон. Однако в большинстве случаев контуры имеют форму многоугольников, имеющих более четырёх сторон. По этой причине предлагается использовать другой метод. Его суть заключается в том, чтобы посчитать протяжённость контура по четырём направлениям: горизонтальное, вертикальное и два диагональных. Для этого необходимо сначала высчитать геометрический центр фигуры (как возможный вариант, его можно посчитать как геометрический центр середин отрезков, входящих в контур). После чего посчитать максимальное расстояние между проекцией контура на каждое из направлений. В данном случае достаточно найти наиболее удалённые проекции концов отрезков и посчитать расстояние между ними по данному направлению. Если хотя бы для одного из направлений получившаяся величина меньше, чем заданный порог, то данный контур отбраковывается.

Пороговое значение, использованное для фильтрации, в общем случае зависит от масштаба съёмки и желаемых результатов. Для того чтобы исключить первый элемент вариации порога, лучше задавать его в метрах и при расчётах переводить в пиксели исходя из масштаба съёмки. Эта информация 
доступна для любых аэрофотоснимков, сделанных для последующей обработки алгоритмами фотограмметрии.

\section{7. Результаты и выводы}

На рис. 2 представлено исходное изображение, полученное в ходе аэросъёмки. К этому изображению мы применили предложенный метод. Результаты выделения областей представлены на рис. 3.

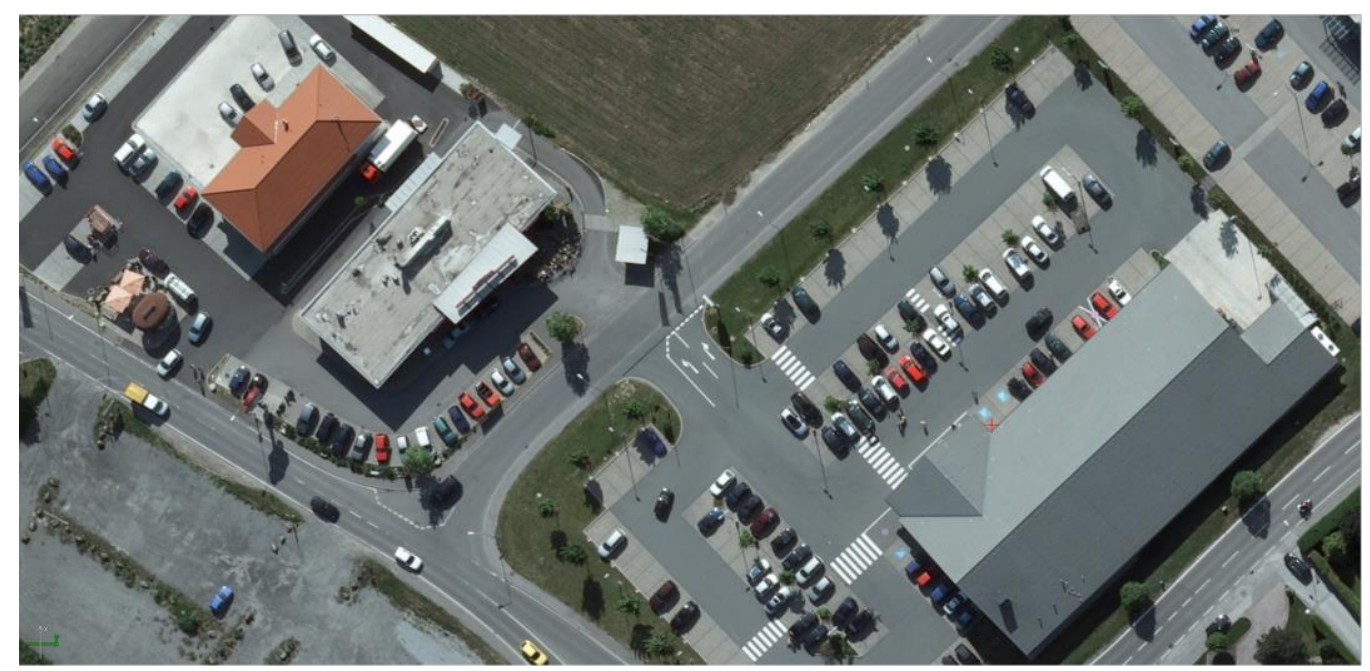

Рис. 2. Исходное изображение

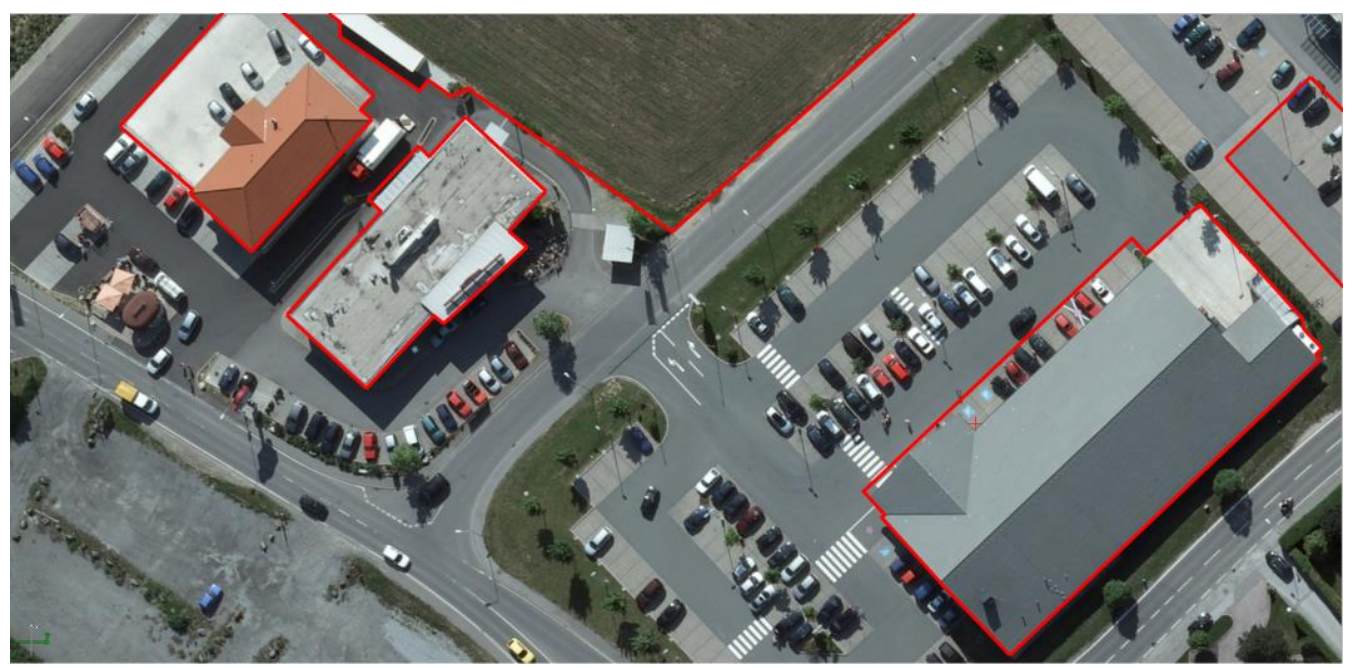

Рис. 3. Изображение с выделенными областями

Средняя точность обнаружения зданий представленным алгоритмом достаточно мала: около $71 \%$. Но поскольку этот метод разработан как предобработка снимков для более аккуратного, но и более ресурсозатратного алгоритма, обрабатывающего снимки снятых с разных ракурсов, то это не так важно. Дело в том, что если на одном снимке строение не обнаружено, то оно будет обнаружено на другом, снятом с более удобного ракурса. Таким образом, точность обнаружения будет примерно такой же, как и с аккуратным 
алгоритмом (к примеру [14]). При этом количество данных, обрабатываемых этим алгоритмом, будет значительно меньше. На аэрофотоснимках описанный в статье метод выделял 20\%-60\% от общей площади в зависимости от типа местности. Это позволяет рассчитывать на ускорение порядка 30\%-60\%, поскольку данные, полученные на всех этапах нашего метода, кроме анализа полученных отрезков и соединения их в контуры, используются потом в алгоритме реконструкции зданий по набору аэрофотоснимков. Дополнительные вычислительные затраты малы, поскольку обработка векторных данных вычислительно проще, чем анализ растров.

Алгоритм, приведённый в данной статье, решает задачу выделения зон, где могут располагаться строения, на основе поиска и анализа замкнутых многоугольников, построенных из множества отрезков. Этот алгоритм может быть использован как самостоятельный метод поиска строений. Однако результаты такого применения заметно уступают более аккуратным, но и более затратным методам [5], [7], [8]. С другой стороны, применение описанного алгоритма в качестве предобработки изображений для последующей передачи полученных результатов алгоритму, обрабатывающему группу изображений сцены [14], существенно повышает эффективность решения глобальной задачи распознавания зданий на аэрофотоснимках.

\section{Список литературы}

1. Brunn A., Weidner U. Extracting buildings from digital surface models. International Archives of Photogrammetry and Remote Sensing, 32(3) sect. 4W2, 1997, pp. 27-34. URL: http://www.ifp.uni-stuttgart.de/publications/wg34/ wg34 brunn.pdf

2. Cord M., Paparoditis N. Jordan M. Dense, reliable and depth discontinuity preserving DEM computation from HRV urban stereopairs. International Archives of Photogrammetry and Remote Sensing, 32, 1997, pp. 49-56.

3. Girard S. et al. Building detection from high-resolution color images. Remote Sensing - International Society for Optics and Photonics, 1998, pp. 278-289.

4. Berthod M. et al. High-resolution stereo for the detection of buildings. Automatic Extraction of Man-Made Objects from Aerial and Space Images, Birkhäuser Basel, 1995, pp. 135-144.

5. Ghaffarian S., Ghaffarian S. Automatic building detection based on supervised classification using high resolution Google Earth images. The International Archives of the Photogrammetry, Remote Sensing and Spatial Information Sciences, 40(3), 2014, pp. 101-106. URL: http://www.int-arch-photogramm-remote-sens-spatial-infsci.net/XL-3/101/2014/isprsarchives-XL-3-101-2014.pdf

6. Ok A. O. Automated Extraction of Buildings and Roads in a Graph Partitioning Framework. ISPRS Annals of Photogrammetry, Remote Sensing and Spatial Information Sciences II-3/W3, 2013, pp. 79-84. URL: http://www.isprs-annphotogramm-remote-sens-spatial-inf-sci.net/II-3-W3/79/2013/isprsannals-II-3-W379-2013.pdf 
7. Jin X., Davis C. H. Automated building extraction from high-resolution satellite imagery in urban areas using structural, contextual, and spectral information. EURASIP Journal on Applied Signal Processing, 2005, pp. 2196-2206.

8. Singhal S., Radhika S. Automatic Detection of Buildings from Aerial Images Using Color Invariant Features and Canny Edge Detection. International Journal of Engineering Trends and Technology(IJETT) 11(8), 2014, pp. 393-396. URL: http://ijettjournal.org/volume-11/number-8/IJETT-V11P277.pdf

9. I. Sobel and G. Feldman. A $3 \times 3$ Isotropic Gradient Operator for Image Processing. R. Duda and P. Hart (Eds.), Pattern Classification and Scene Analysis, 1973, pp. 271-272.

10. Canny J. A computational approach to edge detection. IEEE Transactions on Pattern Analysis and Machine Intelligence, 6, 1986, pp. 679-698. URL: http://cmp.felk.cvut.cz/ cernyad2/TextCaptchaPdf/A\%20Computational\%20Approac h\%20to\%20Edge\%20Detection.pdf

11. Hough P. V. C. Method and Means for Recognizing Complex Patterns, U.S. Patent 3069654, Dec. 18, 1962.

12. Rottensteiner F., Briese C. A new method for building extraction in urban areas from high-resolution LIDAR data. International Archives of Photogrammetry Remote Sensing and Spatial Information Sciences, 34(3/A), 2002, pp. 295-301. URL: http://citeseerx.ist.psu.edu/viewdoc/download?doi=10.1.1.222.428\&rep=rep1\&type= pdf

13. Sohn G., Dowman I. Data fusion of high-resolution satellite imagery and LIDAR data for automatic building extraction. ISPRS Journal of Photogrammetry and Remote Sensing, 62(1), 2007, pp. 43-63. URL: http://citeseerx.ist.psu.edu /viewdoc/download?doi=10.1.1.465.8100\&rep=rep1\&type=pdf

14. Baillard C., Zisserman A. A plane-sweep strategy for the 3D reconstruction of buildings from multiple images. International Archives of Photogrammetry and Remote Sensing, 33(B2), part 2, 2000, pp. 56-62. URL: http://www.isprs.org/proceedings/Xxxiii/congress/part4/23_XXXIII-part4s.pdf

15. Du, S., van Wyk, B. J., Tu, C., \& Zhang, X. An improved Hough transform neighborhood map for straight line segments. IEEE Transactions on Image Processing, 19(3), 2010, pp. 573-585.

16. Li H., Lavin M. A., Le Master R. J. Fast Hough transform: A hierarchical approach. Computer Vision, Graphics, and Image Processing, 36(2), 1986, pp. 139-161.

17. Nguyen T. T., Pham X. D, and Jeon J. An improvement of the Standard Hough Transform to detect line segments. IEEE International Conference on Industrial Technology, 2008, pp. 1-6. URL: https://www.researchgate.net/profile /Thuy_Nguyen38/publication/224327859_An_improvement_of the_Standard_Houg

h_Transform_to_detect_line_segments/links/02e7e51c260e94c03e000000.pdf

18. O'Rourke J. Dynamically Quantized Spaces for Focusing the Hough Transform. IJCAI, 81, 1981, pp. 24-28. 